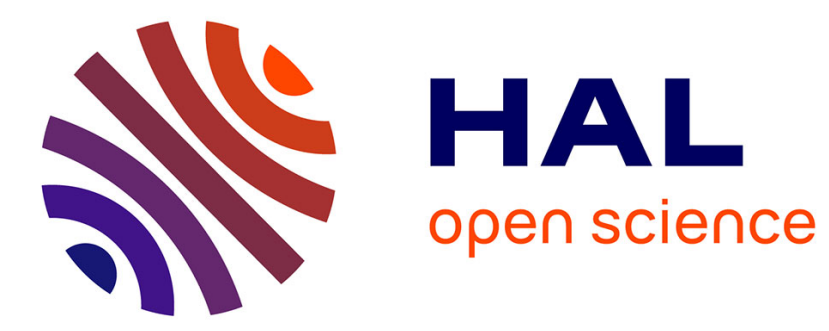

\title{
Femmes lydiennes et crises dynastiques
}

Annalisa Paradiso

\section{To cite this version:}

Annalisa Paradiso. Femmes lydiennes et crises dynastiques. Archimède: archéologie et histoire ancienne, 2018, Archimède n5. Archéologie et histoire ancienne, 5, pp.145-153. halshs-01826371

\section{HAL Id: halshs-01826371 https://shs.hal.science/halshs-01826371}

Submitted on 29 Jun 2018

HAL is a multi-disciplinary open access archive for the deposit and dissemination of scientific research documents, whether they are published or not. The documents may come from teaching and research institutions in France or abroad, or from public or private research centers.
L'archive ouverte pluridisciplinaire HAL, est destinée au dépôt et à la diffusion de documents scientifiques de niveau recherche, publiés ou non, émanant des établissements d'enseignement et de recherche français ou étrangers, des laboratoires publics ou privés. 


\section{ARCHIMĖDE N'5

1 DOSSIER THÉMATIQUE : HUMOEROTICA

ACTUALITÉ DE LA RECHERCHE : DES FEMMES PUBLIQUES. GENRE, VISIBILITÉ ET SOCIABILITÉ DANS L’ANTIQUITÉ GRECQUE ET ROMAINE

108 Marie AUGIER

Des femmes en capacité d'agir. Introduction au dossier

113 Louise BRUIT ZAIDMAN

«L'enfant du foyer ». Des statues pour les filles et les garçons initiés à Éleusis au nom de la cité

124 Hélène CASTELLI

Pèlerines à Épidaure. Femmes, guérison et publicité dans un sanctuaire panhellénique au IV siècle av. J.-C.

134 Marie-Laure SRONEK

Des femmes invisibles dans l'Athènes classique ? Les effets du travail pour une redéfinition de la place des femmes dans la vie publique

\section{Annalisa PARADISO}

Femmes lydiennes et crises dynastiques

\section{Sandra PÉRÉ-NOGUÈS}

Sur les traces de Philistis, « reine » de Syracuse : quelques réflexions sur la visibilité des femmes dans les sources monétaires

165 Christine HUE-ARCÉ

Grec(que)s contre Égyptien(ne)s dans les enteuxeis ptolémaïques : la question du genre dans les $P$. Enteux. 79 et $P$. Enteux. 82

175 Claudia BELTRÃO and Patricia HORVAT

The Name of the Vestal, or When a Vestal is Named

185 VARIA

246 LA CHRONIQUE D'ARCHIMÈDE 


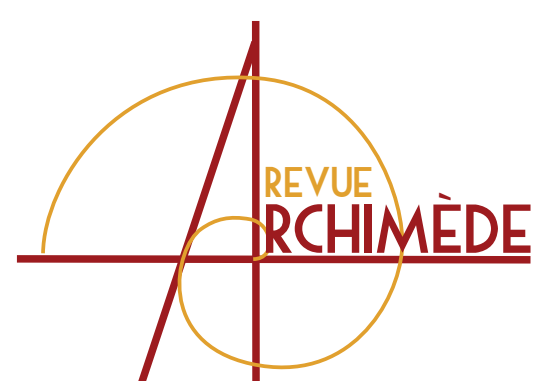

ARCHÉOLOGIE ET HISTOIRE ANCIENNE

\section{FEMMES LYDIENNES ET CRISES DYNASTIQUES}

\section{Annalisa PARADISO}

Maîtresse de conférence en histoire grecque Université de la Basilicate - DICEM Matera

annalisa.paradiso@unibas.it

\section{RÉSUMÉ}

Les femmes lydiennes que nous connaissons par leurs noms sont en général des femmes de condition royale. Dans les sources anciennes, et notamment chez Xanthos de Lydie, quelques-unes de ces femmes jouent un rôle politique, directement

MotS-CLÉs

Lydie,

Hérodote,

Xanthos de Lydie,

Camblès,

Damonnô,

Ardys,

Toudô,

Gygès,

Nanis,

Crésus. ou indirectement, lors de tous les renversements ou les crises des trois dynasties lydiennes que I'historiographie grecque connaît, celles des Atyades, des Héraclides et des Mermnades.
Lydian women whose names we know are royal women. In the ancient sources, especially in Xanthos of Lydia, some of these women play a role that is directly or indirectly political during all the reversals or crises of the three Lydian dynasties that are known to Greek historiography: the Atyad dynasty, the Heraclid and the Mermnad ones.

\section{KEYWORDS}

Lydia, Herodotus, Xanthus of Lydia, Kambles, Damonno, Ardys, Toudo, Gyges, Nanis, Croesus. 
Les sources grecques anciennes présentent les femmes lydiennes essentiellement de deux façons, toutes deux évidentes : les femmes du peuple en groupes, en masse anonyme, et les femmes royales individuellement [1]. Parmi les premières, les sources mentionnent les jeunes filles et leur coutume de se prostituer avant le mariage, afin de constituer leur dot elles-mêmes [2]. Elles évoquent également les concubines royales « eunuchisées »[3], ainsi que les courtisanes qui contribuèrent à la construction du monument funèbre d'Alyatte [4]. Elles décrivent enfin les lamentations funèbres de la foule de femmes lydiennes accourues voir Crésus sur le bûcher [5]. Néanmoins, la plupart des Lydiennes mentionnées par les sources sont des femmes de rang royal, les seules qui ont droit à un nom, en dehors de quelques personnages mythiques comme Omphale ou son esclave Malis, toutes les deux liées à Héraclès dans la tradition [6]. Ce sont les épouses, les mères, les sœurs et les filles de quelques rois lydiens. Par leur lien étroit au pouvoir et la valeur d'échange qu'elles représentent en tant que femmes - en un mot, par leur fonction de femmes royales [7] - elles apparaissent dans le récit comme des protagonistes

[1] Au moins une esclave est individualisée : c'est la servante qui prévient Gygès de l'intention, manifestée par Sadyatte, de le supprimer. Cf. Nicolas de Damas, FGrH $90 \mathrm{~F} 47$ § $8=$ Xanthos de Lydie, BNJ 765 F 37 § 8 . Sur la dépendance de Xanthos de la part du Damascène en ce qui concerne l'histoire lydienne, voir JACOBY, Kommentar à $\mathrm{FGrH} 90 \mathrm{~F} 71$ : « aber N.s quelle für alles lydisches ist Xanthos ».

[2] Hérodote, I, 94. Sur la prostitution des filles, voir PARADISO 2012.

[3] Xanthos de Lydie, FGrH 765 F4ab (= Xanthos, BNJ $765 \mathrm{~F} 4 \mathrm{ab}$ ). Il attribue I'invention de l'eunuchisation à Gygès ou bien au roi Adramytès.

[4] Hérodote, I, 93.

[5] Nicolas de Damas, FGrH 90 F68 § 3 = Xanthos de Lydie, BNJ 765 F43* § 3. tantôt actives ou demi-actives, tantôt passives, et agissent ouvertement ou en coulisses et même malgré elles. C'est ainsi que nous connaissons le nom d'Aryènis, la fille d'Alyatte et sœur de Crésus qui fut donnée en mariage à Astyage, le fils du roi mède Cyaxare, afin de sceller l'accord signé par les Mèdes et les Lydiens à la fin de la guerre qui les opposa [8]. C'est pour la même raison que nous a été transmis le nom de Lydè, la veuve du Mermnade Sadyatte qui apprivoisa par sa douceur leur fils Alyatte, un jeune homme violent envers les nobles lydiens, en l'aidant ainsi à ne pas perdre son pouvoir [9]

Les femmes de rang royal présentent une spécificité : elles émergent dans l'écriture de I'histoire lors de tous les tournants dynastiques ou les crises politiques du pays et elles agissent souvent par le biais de leur sexualité. Parfois elles provoquent la crise ; d'autres fois, elles jouent un rôle tout à fait passif, mais elles participent tout de même aux renversements du pouvoir, aux usurpations et aux ruptures dynastiques. Cela se passe en plein accord avec la réflexion grecque qui attribuait souvent aux femmes un rôle dans la genèse des événements historiques [10]. À cet égard, on peut noter que

[6] Sur Omphale et Alcée, fils d'Héraclès, voir Suda a 1272, qui remonte probablement à Nicolas de Damas et finalement à Xanthos (PARADISO 2015). Sur Malis, Héraclès et leur fils Acelus, voir Hellanicos, FGrH/BNJ 4 F $112=28$ Ambaglio. Pour Hérodote, I, 7, Héraclès eut Alcée d'une esclave anonyme de Iardanos.

[7] Sur la «fonction » des femmes anciennes, voir les contributions rassemblées dans BOEHRINGER \& SEBillotTE-CuCHET 2013.

[8] Hérodote, I, 74.

[9] Xenophilos, BNJ 767 F 1. Voir aussi Nicolas de Damas, FGrH 90 F 64 = Xanthos de Lydie, BNJ 765 F 40.

[10] Voir, par exemple, Hérodote, I, 1-5 qui analyse le rôle (des enlèvements) des femmes dans les responsabilités historiques à l'origine de l'inimitié entre Grecs et barbares. 
le rôle des femmes dans le passage du pouvoir, notamment d'un membre à l'autre de la famille royale, trouve des parallèles historiques importants dans plusieurs systèmes monarchiques. En effet, la spécificité lydienne n'est pas historique dans son ensemble, elle est purement historiographique et consiste dans la répétition, selon un schéma fixe, du rôle décisif, actif ou passif, qui est attribué aux femmes, de façon variée selon les sources, mais systématique dans leur ensemble, lors de toutes les crises de pouvoir. Dans ces récits historiographiques, penser à une femme royale, c'est souvent penser son rôle dans un tournant politique du pays, tandis que penser à une crise politique, c'est toujours penser à la femme royale qui la déclenche.

\section{HÉRODOTE ET LA FEMME ANONYME DE CANDAULE ET DE GYGĖS}

Les pratiques des sources sont néanmoins modulées. Hérodote évoque la succession de trois dynasties en Lydie : la dynastie des Atyades, celle des Héraclides et finalement celle des Mermnades [11], mais l'espace historique qu'il choisit d'analyser dans son Enquête (les trois générations avant les guerres médiques, de 570/560 à 479/478 av. J.-C.) I'amène à s'occuper principalement de I'histoire du Mermnade Crésus, le dernier roi du royaume indépendant [12] dont il relate le rapport avec les Grecs, la tragédie familiale, la dévotion envers les dieux, et finalement la guerre menée contre Cyrus, perdue en 547/546 après avoir mal interprété un oracle de Delphes qui avait annoncé la destruction d'un grand empire, si Crésus avait entamé le conflit [13]. À partir d'une source delphique qui essaie de disculper l'oracle, Hérodote explique I'origine de la chute de Crésus par la faute commise par son ancêtre Gygès, qui tua I'Héraclide Candaule et lui usurpa le trône [14]. C'est à l'intérieur de ce cadre historique et de ces priorités historiographiques qu'Hérodote s'intéresse au changement dynastique des Héraclides aux Mermnades. Son récit, très célèbre, attribue un rôle fondamental et même actif à la femme anonyme de Candaule, tandis qu'il donne beaucoup moins d'importance aux raisons politiques qu'il évoque pourtant mais brièvement [15]. Dès qu'elle entrevoit Gygès, caché derrière la porte de sa chambre, la reine simule une attitude normale mais décide sur-le-champ de se

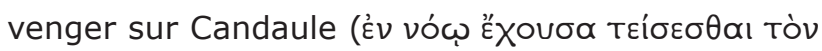

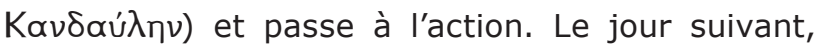
elle convoque (દ́kó $\lambda \varepsilon \varepsilon)$ Gygès et le force à choisir

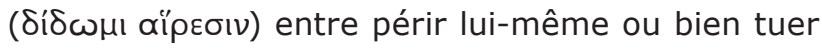
Candaule, accéder à la royauté et l'épouser. Une fois qu'elle a obtenu l'accord de Gygès, la reine organise le meurtre elle-même, en agissant avec décision et rapidité en parfaite conspiratrice : elle dicte les modalités de l'attaque (dans la même chambre, pendant la nuit), s'accorde avec I'homme

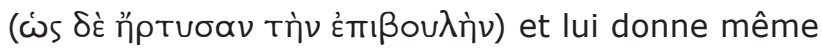

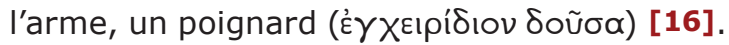

Hérodote n'adopte toutefois pas le même motif lorsqu'il fait une allusion rapide, dans un bref excursus, au changement dynastique des Atyades aux Héraclides qui se produit en vertu d'un oracle, donc apparemment d'une façon paisible [17]. D'ailleurs, même si I'oracle met fin à un conflit, Hérodote choisit de ne pas le rapporter.

\section{XANTHOS DE LYDIE ET LA FEMME ANONYME DE CAMBLÈS}

Une autre source, grecque et contemporaine d'Hérodote, fait un autre choix historiographique et transmet une version noire, radicalement différente de celle esquissée par Hérodote [18]. Pour Xanthos de Lydie, auteur de Lydiaka, le passage des Atyades aux Héraclides ne se passe pas de manière aussi paisible que semble le suggérer Hérodote. Au contraire, les deux dynasties semblent engager une lutte de pouvoir acharnée et une femme anonyme y joue un rôle : il s'agit de l'épouse du roi atyade Camblès. Xanthos, cité par Athénée de Naucratis [19], présente Camblès comme un gros mangeur et un grand buveur : en un mot, un glouton. Une nuit, cet homme dépeça sa femme et la dévora. Le lendemain matin, quand il découvrit la main de celle-ci dans sa bouche, il s'égorgea. Dans la présentation
[11] Hérodote, I, 7 et 14.

[12] MUSTI 1979, p. XVII ; VANNICELLI 1993, p. 19.

[13] Hérodote, I, 6-94 et, sur I'oracle, I, 53.

[14] Hérodote, I, 13, avec FLoWER 1991.

[15] Hérodote, I, 13. Voir PARMENTIER 1995.

[16] Hérodote, I, 10-11.
[17] Hérodote, I, 7.

[18] Selon Éphore, Xanthos est un contemporain plus âgé qu'Hérodote : voir Xanthos de Lydie, FGrH 765 T 5 (BNJ 765 T 5) = Éphore, FGrH/BNJ 70 F 180.

[19] Xanthos de Lydie, FGrH 765 F 18 (= Xanthos, BNJ $765 \mathrm{~F} \mathrm{18a)}$ = Athénée, $\mathrm{X}, 8,415 \mathrm{~cd}$. 
d'Athénée, il ne semble s'agir que de l'histoire d'un meurtre cruel, commis sur un conjoint pour vivre une expérience gastronomique anormale. Mais, quatre siècles plus tard, Nicolas de Damas offre davantage de détails : il affirme l'innocence du roi suicidaire et met I'histoire en relation avec la chute des Atyades. Il attribue en effet la responsabilité de l'événement à Iardanos qui, dans la tradition, est

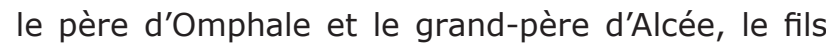
d'Omphale et d'Héraclès qui va devenir l'ancêtre des Héraclides [20]. Autour de Camblès et du meurtre de sa femme, Athénée cite Xanthos. Nicolas de Damas relate la même histoire mais ne cite pas sa source qui est néanmoins Xanthos, en raison des nombreuses correspondances, même littérales, qui relient les deux textes. Nicolas connaît une version différente, plus riche en détails, mais ces différences ne dépendent pas, à mon avis, d'un filtre hellénistique qu'aurait transmis Xanthos à Nicolas [21], ni d'une réélaboration de la part de ce dernier [22], mais des différents critères de sélection adoptés par Athénée et Nicolas en citant et résumant leur source commune Xanthos. Chez Nicolas de Damas, Camblitas (et non Camblès) se proclame innocent et pense qu'il a agi sous l'effet d'une drogue. Chez

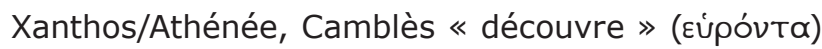
la main de sa femme dans sa bouche le lendemain du crime : s'il ne prend conscience de son acte qu'à l'aube, c'est qu'il a évidemment agi pendent la nuit sous l'effet de quelques éléments externes (une drogue?), donc il est, dans un certain sens, innocent. Par conséquent, Xanthos aussi présentait le meurtre de la femme comme un homicide involontaire [23]. L'histoire a un antécédent et une suite dans la lutte engagée entre Camblitas et Iardanos : I'allusion à la « haine » de Iardanos laisse entendre que celui-ci se venge de quelques méfaits commis par Camblès/ Camblitas. Le conflit entre les deux personnages est décidé par la mort de l'épouse de Camblès, qui entraîne la suite des événements, donc le suicide du roi et, vraisemblablement, la prise du pouvoir par les Héraclides. Le renversement se produit donc avec le meurtre puis le dépeçage et l'acte de dévorer le corps de la femme-victime du roi, qui devient la protagoniste tout à fait involontaire du changement dynastique. Anonyme, sujet passif, la femme de Camblès ne figure même pas dans les réactions du lendemain, ni celle de son mari, qui se disculpe par son suicide, ni celles des Lydiens, qui s'interrogent sur les raisons du meurtre.

\section{XANTHOS ET DAMONNÔ, LA FEMME DE KADYS}

Le motif de la rupture violente et du rôle pivot d'une femme est repris par Xanthos à plusieurs reprises. Il I'applique en effet, de façon systématique, à tous les tournants dynastiques et politiques du pays, dont il rédige une histoire complète et détaillée. Pendant le long règne des Héraclides, c'est encore une femme de condition royale qui entraîne, cette fois activement, une crise « intra-dynastique ». Damonnô, la femme du roi héraclide Kadys, décide d'éliminer son époux en plein accord avec son amant Spermos,

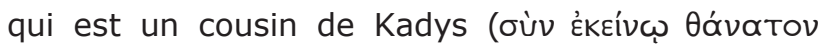

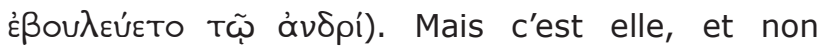
Spermos, qui passe à l'acte, acte criminel, politique et constitutionnel : elle donne à Kadys du poison

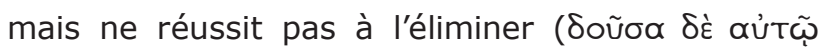

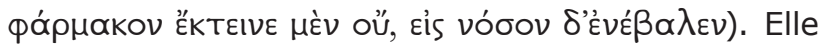
organise ensuite le meurtre du médecin qui a soigné Kadys avec succès : elle fait creuser un trou dans la terre qu'elle cache par des lits. Ensuite, elle invite le médecin à dîner et le fait tomber dans le trou qu'elle fait couvrir avec de la terre [24]. Finalement, Kadys meurt et Damonnô, avec son amant, exile le frère de son époux, Ardys, corrompt beaucoup de Lydiens, épouse Spermos et assure elle-même officiellement la transmission du pouvoir en proclamant roi son nouveau mari [25].

Damonnô joue la même fonction que la femme de Camblès, mais contrairement à elle, son rôle est actif. Elle est donc une figure d'autorité et de pouvoir politique et constitutionnel. Ce pouvoir est même inscrit dans son nom, qu'il évoque le titre hittite de la
[20] Nicolas de Damas, FGrH 90 F 22 = Excerpta de virtutibus et vitiis I p.339, 5. Sur Omphale, Héraclès et Alcée, voir, supra, n. 6.

[21] RegenBOGEN 1943, p. 21; Diller 1956, p. $74=$ NeWiger \& Seyffert 1971, p. 459.

[22] TOHER 1989, p. 169.

[23] Voir à ce propos, avec davantage de détails, Xanthos de Lydie, Commentary on BNJ 765 F 18b.

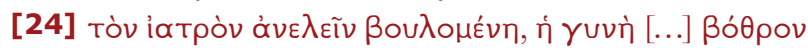

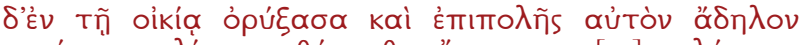

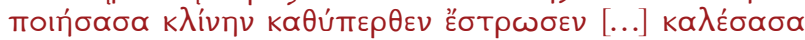

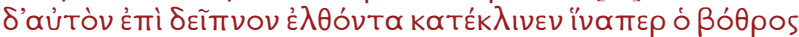

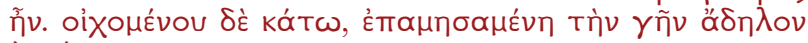

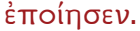

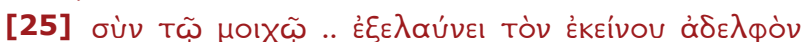

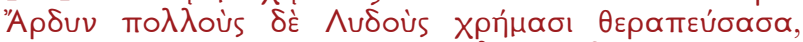

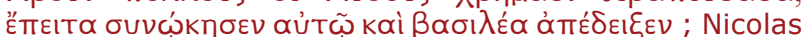
de Damas, FGrH 90 F 44 §§ 2-3 = Xanthos de Lydie, BNJ F 34a §§ 2-3. 
reine (tawananna [26]), ou qu'il représente l'hypocoristique grec d'un $* \Delta \bar{\alpha} \mu o-v i ́ k \alpha[27]$. Le récit pourrait avoir un arrière-plan historique, mais le personnage de Damonnô est à mettre en relation avec une tradition littéraire mixte, grecque et anatolienne. Elle est en quelque sorte une Clytemnestre lydienne qui agit également en tant que femme adultère. Dans I'Agamemnon d'Eschyle, Clytemnestre tue son mari Agamemnon (v. 1372 sqq., ayant été séduite par un cousin du roi, Égisthe, qui a ourdi le crime (v. 1604) et va régner jusqu'à la vengeance d'Oreste ( $v$. 16381639). De la même façon, Damonnô tue son mari elle-même, après avoir été séduite par un cousin à lui, qui va régner pendant deux ans, avant de perdre la vie. En outre Damonnô s'inscrit dans une série de représentations grecques de femmes de pouvoir anatoliennes, et notamment lydiennes. Si on laisse de côté son adultère, elle est un double, quant à la fonction, de la femme de Candaule. Son personnage s'inspire évidemment aussi d'Omphale, la reine mythique de Lydie, dont l'image de femme dominatrice et de maîtresse d'Héraclès est déjà présente à l'époque de Xanthos (vraisemblablement actif pendant les années 50 et 40 ) et suggère des comparaisons malicieuses avec Aspasie. Vers la moitié du ve siècle, le mythe d'Omphale et d'Héraclès est en effet populaire, car il est introduit au théâtre dans la tragédie (chez l'Agamemnon d'Eschyle, les Trachiniennes de Sophocle) et le drame satyrique (chez l'Omphale d'Ion de Chios et celui d'Achée d'Érétrie) ; entre le ve et le IV siècle, il figure aussi dans la comédie (chez Archippos, Nicocharès, Antiphane, Cratinus, mais il n'est pas sûr qu'il soit attesté déjà chez Cratinos ou bien Eupolis) [28].

[26] KARWIESE 1991, p. 10 n. 40 ; OETTINGER 1995, p. 43. [27] NEUMANN 1974, p. 185-186.

[28] Eschyle, Agamemnon, 1040-1 ; Sophocle, Les Trachiniennes, 69-71, 252-257 et 356-357 ; Ion de Chios, TGrF I 19 frr. 17a-33a et 59 (= frr. 22-28 Leurini²); Achée TGrF I 20 frr. 32-35; Archippos frr. 8-13 K.-A. ; Nicocharès fr. 7 K.-A ; Antiphane frr. 174-175 K.-A. ; Cratinus fr. 5 K.A.; fr. adesp. 704 K.-A. Quant à Cratinos fr. $* 259$ K.-A. et Eupolis fr. 294 K.-A., la scholie à Platon, Ménéxène, 235c Greene qui les transmet présente un texte corrompu qui

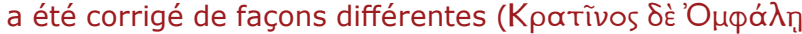

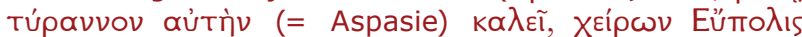

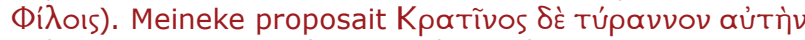

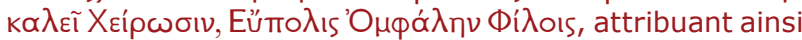
la définition d'Aspasie comme «tyran » à Cratinos et la comparaison entre Aspasie et Omphale à Eupolis : voir aussi Cratinos fr. *259 K.-A. SCHWARZE 1971, p. 58-59 n'a corrigé par contre que $X \varepsilon i \rho \omega v[a]$ et a accepté, dans le texte, la citation de I'Omphale de Cratinus. Sur Omphale au ve siècle, voir aussi Phérécyde, FGrH/BNJ $3 \mathrm{~F}$ 82b et Hérodore, FGrH/BNJ $31 \mathrm{~F} 33$ et 41ab.

\section{XANTHOS ET TOUDÔ, LA FEMME DE SADYATTE ET DE GYGÈS}

Avec I'histoire de l'épouse du dernier des Héraclides, Xanthos offre une variante de la version correspondante d'Hérodote et en même temps construit un double de son propre récit de la crise « intra-dynastique » des Héraclides. Chez Hérodote, l'épouse anonyme de Candaule est à l'origine de la mort de son mari et aussi de la chute de la dynastie : tout comme Damonnô, elle transmet le pouvoir à son deuxième époux. Sa fonction dans l'économie de l'histoire est celle-ci et le motif érotique n'intervient pas [29], car elle n'est pas amoureuse de Gygès mais veut simplement se venger de Candaule. Gygès n'est pas amoureux d'elle non plus. Seul Candaule est pris d'un excès d'amour pour sa femme. Xanthos en revanche, qui puise dans des traditions semblables mais pas identiques à celles d'Hérodote, introduit quelques modifications, concernant le rôle de la femme et le motif érotique, dans cette intrigue où un usurpateur tue son prédécesseur et en épouse la femme [30]. L'épouse de Candaule, anonyme chez Hérodote, s'appelle Toudô chez Xanthos/Nicolas de Damas [31]. Toudô revêt la même fonction que la femme de Candaule, mais n'a pas le même rôle : en provoquant indirectement la crise dynastique, elle joue en effet un rôle demi-actif. Elle repousse résolument les avances de Gygès et accuse le jeune homme auprès de son mari Sadyatte, mais ne provoque pas intentionnellement le meurtre de celui-ci [32]. Le motif érotique est présent, mais pas de son côté, ni de celui de Sadyatte. C'est Gygès qui essaye de la séduire

[29] Si non, peut-être, indirectement, s'il est vrai que Gygès fréquentait habituellement la reine : cf. фoıт̃̃ $v$ chez Hérodote, I, 11.

[30] Sur la dépendance de traditions semblables de la part d'Hérodote et de Xanthos, RADET 1893, p. 112, 123-124.

[31] Hesychios interprète TUT'́ comme le nom d'un oiseau, la chouette (cf. T 1692 Hansen-Cunningham,

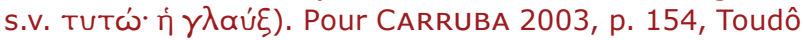
est un hypocoristique anatolien dérivé, par exemple, de Duduhepas or Tuttuwanis. La femme est anonyme aussi chez Platon, République, II, 3, 359d-360a et dans un fragment tragique d'époque inconnue $(P O x y 2382=$ TrGF II, 664).

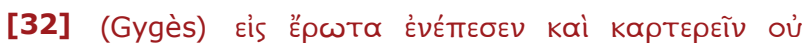

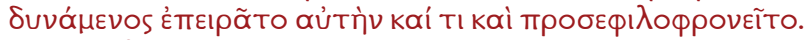

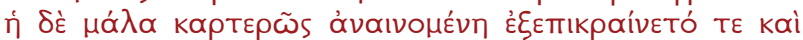

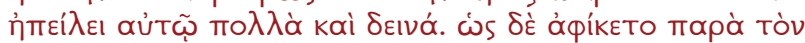

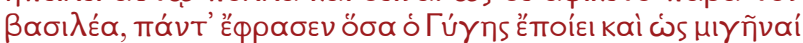

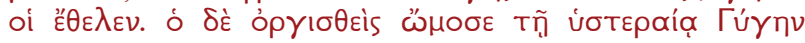

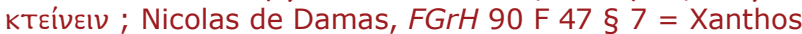
de Lydie, BNJ 765 F 37 § 7. Voir PARMENTIER 1995. 
et c'est donc sa tentative qui déclenche la crise dynastique. Néanmoins, le motif érotique n'est pas la seule explication de la crise : il s'agit en fait d'un prétexte, car la crise fait suite à une ancienne malédiction que le vieux roi Héraclide Ardys (le frère de Kadys) avait lancée contre le meurtrier inconnu de son favori Dascyle, I'ancêtre de Gygès (en réalité, le meurtrier est son propre fils Adyatte, mais Ardys l'ignore) [33]. L'assassin n'ayant pas payé pour son crime, la malédiction reste active et touche son descendant, donc Sadyatte, par la main de Gygès, comme lui-même le rappelle aux amis avec lesquels il a ourdi le complot. Ainsi, le motif érotique est introduit dans une histoire politique plus complexe, qui relie plusieurs générations d'Héraclides par la malédiction lancée par Ardys, malédiction qui trouve son aboutissement dans le meurtre de Sadyatte. Toudô entre dans I'histoire d'un enchaînement de meurtres croisés : celui de l'Héraclide Sadyatte, accompli par le Mermnade Gygès et expié à la cinquième génération par son descendant Crésus (Gygès-Ardys-Sadyatte-Alyatte-Crésus), qui réplique un autre meurtre, celui de Dascyle, le Mermnade ancêtre de Gygès, accompli par I'Héraclide Adyatte et expié quelques générations plus tard par son descendant Sadyatte (Adyatte-Meles(Sadyatte le régent)-Myrsos-Sadyatte) [34].

Xanthos élabore ainsi une généalogie tragique de meurtres et de châtiments, une chaîne qui explique le passage dynastique des Héraclides aux Mermnades et la chute de ces derniers, en doublant le même épisode (un meurtre, expié quelques générations après, qui entraîne un autre meurtre à expier). Ce schéma répété fonctionne comme un outil interprétatif de I'histoire dynastique lydienne et dans ce schéma Xanthos introduit la femme du dernier des Héraclides, Toudô. Aussi, même si le motif du passage du pouvoir à travers une femme royale n'est pas invraisemblable en soi, la répétition du schéma sème des doutes sur l'historicité de ces rôles féminins. De plus, Xanthos - et lui seulement
- multiplie les personnages féminins ayant un rôle spécifique dans toutes les crises politiques, « intra »- et « inter-dynastiques ». Au sommet de la chaîne des meurtres, Xanthos attribue un rôle pivot même à Dascyle, qui est un double de la femme de Camblès, de Damonnô et de Toudô, mais aussi de Sadyatte/Candaule, car il est d'un côté le favori

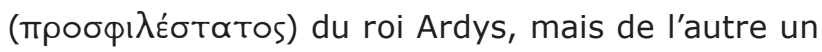
homme puissant, un presque usurpateur qui provoque la jalousie de l'héritier légitime. Son assassinat par celui-ci déclenche la chaîne des meurtres qui ne s'achève qu'avec la chute de Crésus, donc avec la fin du royaume indépendant [35].

\section{PARTHÉNIUS ET NANIS, LA FILLE DE CRÉSUS}

Le texte de Xanthos est transmis en fragments ou à travers les abrégés que les compilateurs byzantins des Excerpta Constantiniana ont tirés de I'Histoire Universelle de Nicolas de Damas. Son histoire de la prise de Sardes n'a pas survécu, donc on ignore de quelle façon il organisait le récit et $s^{\prime}$ il y reprenait ce motif en introduisant des personnages féminins. Mais quatre siècles plus tard, nous le retrouvons dans une réécriture tardive de cette histoire. Si dans I'historiographie classique, chez Hérodote, Xénophon et Ctésias [36], la prise de la ville est inscrite dans une logique purement militaire, Parthénius au $\mathrm{I}^{\mathrm{er}}$ siècle av. J.-C. relate une version isolée, d'après laquelle l'acropole de Sardes fut prise par Cyrus grâce à la fille de Crésus, nommée Nanis. Celle-ci fit un pacte secret avec Cyrus et s'engagea à trahir sa ville à condition qu'il la prenne comme épouse [37]. Nanis intro-

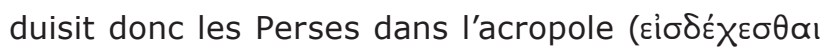
Toùs חо $\lambda \varepsilon \mu$ íous). Quant à Cyrus, il conquit la ville mais ne tint pas sa promesse [38]. L'histoire de la fille de Crésus s'insère dans le motif bien connu de la trahison des liens de sang par amour [39].
[33] PARMENTIER 1995.

[34] Pour la succession de cinq rois Mermnades (GygèsArdys-Sadyatte-Alyatte-Crésus), cf. Hérodote, I, 6-16 ; pour la succession de quatre rois Héraclides (AdyatteMeles-Myrsos-Sadyatte : Sadyatte le régent est un Tylonide), voir Nicolas de Damas, FGrH 90 FF 44-47 = Xanthos de Lydie, BNJ 765 FF 34a-37.

[35] Nicolas de Damas, FGrH 90 F 44a $\S 11=$ Xanthos de Lydie, BNJ 765 F 34a § 11. Dascyle est un double aussi de Magnes, l'éromène grec de Gygès qui est tué par les Magnésiens, provoquant ainsi la réaction du roi, l'attaque et la conquête de Magnésie du Sipyle : cf. Nicolas de Damas, FGrH 90 F 62 = Xanthos de Lydie, BNJ 765 F 38.

[36] Hérodote I, 84 ; Xénophon, Cyropédie, VII, 2, 1-4 ; Ctésias, FGrH 688 F9 = F9 Lenfant, suivi par Tzetzès, Théon et Polyen (= Ctésias FF9a, 9b, 9c Lenfant).

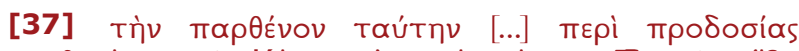

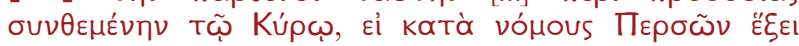

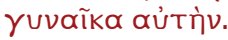

[38] Parthénios, Narrations d'amour, XXII.

[39] TUPLIN 2010. 
Parthénius focalise l'attention sur un personnage féminin, l'érotise et le lance dans la grande histoire de la conquête perse du royaume Iydien en c. 547/546 av. J.-C. [40]. Ses sources sur cette malheureuse passion amoureuse sont Licymnios de Chios (poète lyrique du IV siècle av. J.-C.) et Hermésianax de Colophon (poète élégiaque du IV ${ }^{\mathbf{E}}$ III ${ }^{\mathrm{e}}$ siècle av. J.-C.), dont les noms ont été annotés par un lecteur ancien sur la manchette du Palatinus Heidelbergensis gr. 398 - le manuscrit de la deuxième moitié du IX ${ }^{e}$ siècle ap. J.-C. qui seul transmet les Narrations d'amour [41]. Mais I'histoire remonte au moins à la source de la Chronique romaine de 16-20 ap. J.-C., qui mentionne la trahison par amour de «la fille » de Crésus, sans pourtant en mentionner le nom [42]. Cette source a été identifiée avec Éphore [43]. L'histoire de Nanis, qui porte en effet un nom proprement lydien [44], remonte donc plus loin dans le temps, au IV siècle. Elle étend à I'histoire de Crésus le motif de I'association d'une femme à une crise politique et, dans ce sens, retravaille au moins le texte d'Hérodote si elle n'est pas dérivée de Xanthos. Elle s'inscrit pleinement dans un double processus de réécritures déjà pré-hellénistiques : celle, historique, de la prise de Sardes et celle, biographique, des membres de la famille de Crésus. Bacchylide quant à lui, qui évoque dans l'Épinicie III une épouse et « des filles », au pluriel, de Crésus, présente une famille solidaire du destin du roi, qui décide de se suicider [45]. Ni Hérodote ni Xanthos de Lydie ne s'éloignent de cette perspective, mais les détails peuvent varier. Hérodote relate la mort tragique du fils Atys et la guérison prodigieuse du second fils muet, qui recouvre la parole pour protéger son père de l'attaque d'un Perse [46]. Il suit I'histoire de la famille jusqu'à un descendant de Crésus, Pythios, le fils d'Atys [47]. Xanthos/Nicolas de Damas développe le motif du fils muet qui veut partager le destin de son père sur le bûcher et nomme aussi d'autres enfants qui sont rendus au roi avec « ses

[40] En réalité, avant 539/538 av. J.-C., c'est-à-dire avant la prise de Babylone par Cyrus. Néanmoins, la date de $547 / 546$ a été récemment confirmée par VAN DER SPECK 2014, p. 256 n. 184.

[41] À moins que ses noms fussent des loci paralleli. La définition de manchette appartient à PAPATHOMOPOULOS 1968, p. xi.

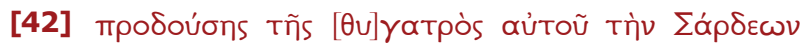

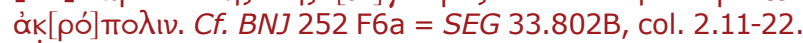

[43] HASLAM 1986, p. 198 et LIGHTFOOT 1999, p. 505.

[44] Cf. ZGUSTA 1964 § 347-8 et, pour Nannaś = Návvas, attesté dans une inscription bilingue, GUSMANI 1964, p. 169-170, 259 no. 20. épouses » (au pluriel) après sa libération [48]. Même chez Xénophon, son épouse et ses filles sont rendues à Crésus [49]. Mais chez Ctésias, la femme de Crésus se tue, en se précipitant du haut du rempart, après la mort de son fils qui a été pris comme otage et que Crésus n'a pas réussi à libérer [50]. Cependant Parthénius et, avant lui, la source de la Chronique romaine (Éphore) brisent la solidarité de ce groupe familial, ouvrant ainsi la voie aux solutions du roman. Ils prêtent à sa fille, Nanis, le rôle de protagoniste négatif et introduisent par conséquent le motif de la trahison motivée par une raison érotique, la passion malheureuse de la jeune fille pour Cyrus. En d'autres termes, ils attribuent à la fille de Crésus cette érotisation qui est niée, dans toutes les sources, à son épouse.

\section{LA FEMME DU DERNIER HÉRACLIDE ET SA FONCTION DANS L'HISTORIOGRAPHIE PLUS TARDIVE}

Les réécritures littéraires plus tardives de I'histoire de Candaule/Sadyatte, de sa femme et de Gygès retravaillent le personnage féminin, tout en déplaçant l'accent de la fonction institutionnelle/ politique vers d'autres identités. Elles s'inspirent partiellement d'un prototype littéraire, la même histoire telle qu'elle est relatée par Platon, où la femme est séduite par Gygès [51]. Ptolémée Héphestion rassemble quant à lui des sources, parfois douteuses et anonymes (seul un certain Abas y est explicitement nommé), qui donnent à la femme de Candaule un éventail de noms : Nysia, Toudous, Clytia et Abrô [52]. Ces noms fictifs transmettent des informations sur l'origine géographique ou bien la moralité du personnage. Nysia semble une coquille pour Mysia (= la femme mysienne) [53], tandis qu'Abrô est un nom érotique : la femme chaste d'Hérodote et de Xanthos, celle qui théorise la nudité comme absence de

[45] Bacchylide, Épinicies, III, 34-35.

[46] Hérodote, I, 34-46 et 85, avec SEBEOK-BRADY 1978 et ALLÉLY 2009.

[47] Hérodote, VII, 27-29 et 38-39 : cf. VANOTTI 2013.

[48] Nicolas de Damas, FGrH 90 F 68 §§ 4-7 et $14=$ Xanthos de Lydie, BNJ 765 F 43* §§ 4-7 et 14.

[49] Xénophon, Cyropédie, VII, 2, 26.

[50] Ctésias, FGrH 688 F 9 = F 9 Lenfant.

[51] Platon, République, II, 3, 359d-360a.

[52] Ptolémée Héphestion chez Photius, Bibliothèque CXC, 150b18-29: Abas, BNJ $46 \mathrm{~F} 2$.

[53] MÜLLER, FHG III, 384. 
pudeur [54] et refuse résolument les avances de Gygès, devient la femme séduite de Platon et finalement prend chez Abas un nom qui fait allusion à I'habrosyne lydienne, le nom d'une courtisane.

Ces réécritures achèvent le processus d'érotisation de la femme de Candaule : avec une toute autre identité, elles lui attribuent aussi un nom différent. Cela contraste avec I'histoire littéraire de Gygès, qui est présenté par les sources comme un homme luxurieux, à l'érotisme insatiable, le seul à l'être parmi les rois lydiens, presque dès le début de son histoire littéraire. L'homme de rupture, I'usurpateur, est raconté à travers sa conquête du pouvoir, qui se complète par le mariage avec la reine, si bien que déjà très tôt il est décrit à travers sa sexualité. La relation de Gygès avec la femme de Candaule, chaste chez Hérodote, prend d'autres significations chez Xanthos qui contribue fortement à fixer l'identité bisexuelle de Gygès. Xanthos lui attribue au moins deux amours outre Toudô : la servante qui lui ouvrit la porte de la chambre de Sadyatte, lui permettant de le tuer, et Magnès, le poète grec philo-lydien qui fut massacré par les Magnésiens [55]. Xanthos lui attribue encore l'invention des femmes-eunuques: il s'agit vraisemblablement du récit lydien de l'invention de la sodomie [56]. Celles-ci sont les sources conceptuelles de Cléarque de Soles, qui interprète Gygès à la lumière d'un critère différent, celui de la truphê lydienne. Cléarque rappelle I'amour inconditionnel que Gygès eut pour sa maîtresse, non seulement pendant sa vie, car il lui avait confié sa personne et son royaume, mais aussi après sa mort, quand il rassembla tous les Lydiens du territoire et leur fit élever le monument nommé « le tombeau de la courtisane », un monument si haut que Gygès, de même que tous les Lydiens, pouvait le voir de partout, quand il voyageait en deçà de la région du Tmolus [57]. Gygès, qui devient « célèbre » à cause d'une courtisane, et lui offre sa personne et son royaume, est rangé par Cléarque à côté de Périclès, ironiquement appelé I'Olympien et ouvertement blâmé pour avoir « troublé la Grèce entière » à cause d'Aspasie, elle aussi une courtisane [58]

[54] Hérodote, I, 8.

[55] Nicolas de Damas, FGrH 90 F 47 § 8 et $62=$ Xanthos de Lydie, BNJ 765 F 37 § 8 et 38.

[56] Xanthos de Lydie, FGrH $765 \mathrm{~F} 4 \mathrm{a}$ et $4 \mathrm{~b}$ (= Xanthos, BNJ $765 \mathrm{~F} 4 \mathrm{a}$ et 4b) : cf. PARADISO 2012 et, pour l'érotisation de Gygès, CAZZANIGA 1953, 396 n. 1.

[57] Cléarque, fr. 29 Wehrli $^{2}=47$ Taïfakos.

[58] Cléarque, fr. 30 Wehrli $^{2}=48$ Taïfakos.

[59] LARSON 2006.

\section{EN GUISE DE CONCLUSION}

Dans I'historiographie grecque qui enquête sur I'histoire dynastique lydienne, la fonction des femmes de condition royale est claire : dans les différents récits, elles jouent un rôle actif (Damonnô, la femme de Candaule, Nanis), demi-actif (Toudô) ou bien passif (la femme de Camblès), mais elles émergent lors de tous les tournants politiques inter- et intra-dynastiques. Leur fonction est donc celle de provoquer, directement ou indirectement, la crise politique : quand elles y jouent un rôle actif ou demi-actif, elles le font en tant que figures d'autorité et de pouvoir politique et (parfois) constitutionnel. Leurs actions impliquent l'élimination du roi légitime ou l'accord avec l'ennemi, mais n'expriment pas une vision politique, n'envisagent que les intérêts privés de ces femmes qui conditionnent finalement la communauté entière. Ces intérêts privés sont centrés sur leur sexualité. Damonnô s'efforce de transmettre le pouvoir royal à son amant et pour obtenir cela n'hésite pas à tuer, à exiler et à corrompre ; la femme du dernier des Héraclides réclame et obtient la vengeance de la pudeur violée, donc une compensation de I'amour-propre blessé ; Nanis trahit sa patrie pour épouser le roi ennemi. Les répercussions politiques de ces actions sont graves : Damonnô transmet le pouvoir à un usurpateur qui ne règne que deux ans et laisse un souvenir très négatif. La femme de Candaule/Sadyatte déclenche un renversement dynastique qui sera puni à la cinquième génération après Gygès, donc à la génération de Crésus : en fait, elle est indirectement à l'origine de la chute de Sardes et de la fin du royaume indépendant. Au contraire, Nanis provoque directement la fin de la dynastie et livre la Lydie aux Perses.

La fonction et le rôle de ces femmes ne se reflètent dans leurs noms que d'une façon irrégulière. Damonnô a un nom, même significatif, mais la femme du dernier Héraclide, nommé Toudô par Xanthos, est anonyme chez Hérodote. Il est difficile de comprendre les raisons de cette absence, étant donné qu'Hérodote et Xanthos sont contemporains et dépendent de traditions semblables : il se peut qu'Hérodote ait choisi de ne pas la mentionner en signe de respect, dans le but de I'« innocenter», ainsi que le croit Stephanie Larson [59], mais ce choix a fini par renforcer le rôle et la fonction de la femme au détriment de son identité individuelle. Ce n'est que dans les sources plus tardives qu'elle reçoit des noms, au pluriel, quand sa fonction n'est plus «politique», 
mais elle est désormais réduite à la dimension érotique du personnage. En tant que protagoniste d'une Narration d'amour de Parthénius, Nanis ne pouvait avoir qu'une identité nominale. Quant à la femme de Camblès, elle est anonyme dans les deux Mittelquellen (Nicolas, Athénée), mais nous ne savons pas si Xanthos connaissait son nom ou a décidé de ne pas le mentionner. Il est possible aussi que ce nom a été perdu dans le processus de sélection que le texte de Xanthos a subi. Quelle qu'en soit la raison, l'absence du nom souligne encore une fois le rôle, ici passif, de la femme, dont la fonction est néanmoins celle, chargée de conséquences, de permettre le passage du pouvoir d'une dynastie à l'autre, des Atyades aux Héraclides.

\section{BIBLIOGRAPHIE}

ALLÉLY, Annie, 2009, «La parole retrouvée du fils de Crésus à travers les sources grecques et latines : étude philologique et anthropologique », Latomus 68, p. 16-34.

Boehringer, Sandra \& Sebillotte-Cuchet, Violaine (éd.), 2013, « Des femmes en action. L'individu et la fonction en Grèce antique », Mètis hors série.

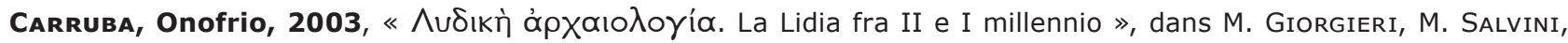
M.-C. Trémouille \& P. VAnnicelli (éd.), Licia e Lidia prima dell'ellenizzazione, Roma, p. 145-169.

Cazzaniga, Ignazio, 1953, «Il frammento tragico di Gige e la tradizione retorica », La Parola del Passato 8, p. 381-398.

Diller, Hans, 1956, «Zwei Erzählungen des Lyders Xanthos », in Navicula Chiloniensis. Studia philologa F. Jacoby octogenario oblata, Leiden, p. 66-78 ( = H.J. Newiger \& H. Seyffert (éd.), H. Diller, Kleine Schriften zur antiken Literatur, München, 1971, p. 451-463).

Flower Harriet I., 1991, « Herodotus and Delphic Traditions about Croesus », dans M.A. Flower \& M. Toher (éd.), Georgica. Greek Studies in Honour of George Cawkwell, London, p. 57-77.

GusmanI, Roberto, 1964, Lydisches Wörterbuch, Heidelberg.

HASLAM, M.W., 1986, «The Fall of Sardis in the Roman Chronicle », Zeitschrift für Papyrologie und Epigraphik 62, p. 198.

KARWIESE, Stefan, 1991, «The Artemisium Coin Hoard and the First Coins of Ephesus », Revue Belge de Numismatique et de Sigillographie 137, p. 1-28.

LARSON, Stephanie, 2006, «Kandaules' Wife, Masistes' Wife: Herodotus' Narrative Strategy in Suppressing Names of Women (Hdt. 1.8-12 and 9.108-13) », The Classical Journal 101, p. 225-244.

LightfOот, Jane, 1999, Parthenius of Nicaea, Oxford.

Mustr, Domenico (éd.), 1979, La storiografia greca. Guida storica e critica, Roma - Bari.

NeumanN, Günter, 1974, «Der Name der lydischen Königin Damonno », Zeitschrift für vergleichende Sprachforschung 88, p. $185-186$.

Oettinger, Norbert, 1995, « Anatolysche Etymologien », Historische Sprachforschung/Historical Linguistics 108, p. 39-49.

Papathomopoulos, Manolis, 1968, Antoninus Liberalis, Les métamorphoses, Paris.

Paradiso, Annalisa, 2012, «Les catalogues des inventions lydiennes», dans V. Azoulay, F. Gherchanoc \& S. Lalanne (éd.), Le banquet de Pauline Schmitt Pantel. Genre, Mœurs et politique dans I'Antiquité grecque et romaine, Paris, p. 131-148.

Paradiso, Annalisa, 2015, «A New Fragment for Nicolaus of Damascus? A Note on Suda a $1272 »$, Histos 9, p. 67-75. Paradiso, Annalisa, 2018, Xanthos of Lydia (765), Brill's New Jacoby.

Parmentier-Morin, Édith, 1995, « La Lydie, Hérodote et Nicolas de Damas », Ktèma 20, p. 85-94.

RADET, Georges, 1893, La Lydie et le monde grec au temps des Mermnades (687-546), Paris.

Regenbogen, Otto, 1943, «Review of L. Pearson, Early Ionian Historians », Gnomon 19, p. 8-26.

Sebeok, Thomas A. \& BRAdy, Erika, 1978, «The Two Sons of Croesus: A Myth About Communication in Herodotus », Journal of the Folklore Institute 15, p. 5-22.

SchwARZE, Joachim, 1971, Die Beurteilung des Perikles durch die attische Komödie und historische und historiographische Bedeutung, München.

TOHER, Mark, 1989, « On the Use of Nicolaus' Historical Fragments », Classical Antiquity 8, p. 159-172.

TUPLIN, Christopher, 2010, Commentary on BNJ $691 \mathrm{~F} * 1$.

VAN DER SPEK, Robert J., 2014, « Cyrus the Great, Exiles, and Foreign Gods: A Comparison of Assyrian and Persian Policies on Subject Nations », dans M. Kozuh, W.F.M. Henkelman, Ch.E. Jones \& Chr. Woods (éd.), Extraction and Control: Studies in Honor of Matthew W. Stolper, Chicago, p. 233-264.

Vannicelli, Pietro, 1993, Erodoto e la storia dell'alto e medio arcaismo (Sparta-Tessaglia-Cirene), Roma.

VANotrI, Gabriella, 2013, «Pythios/Pythes e la moglie da Erodoto a Plutarco », Anabases 18, p. 11-32.

Zgusta, Ladislav, 1964, Kleinasiatische Personennamen, Praha. 